Editorial

Paediatr Paedolog 2018 53 (Suppl 1):S1-S2 https://doi.org/10.1007/s00608-018-0620-3

(c) Springer-Verlag GmbH Austria, ein Teil von Springer Nature 2018

CrossMark

\author{
Christian Popow ${ }^{1,2} \cdot$ Nicole Grois $^{3} \cdot$ Regina Rath-Wacenovsky $^{4} \cdot$ Ferdinand Sator $^{5} \cdot$ \\ Ernst Tatzer ${ }^{6,7}$ \\ ${ }^{1}$ Universitätsklinik für Kinder- und Jugendpsychiatrie, Medizinische Universität Wien, Wien, Österreich \\ ${ }^{2}$ Psychiatrische Abteilung für Kinder- und Jugendpsychiatrie und Psychotherapie, Landesklinikum Mauer, \\ Mauer bei Amstetten, Österreich \\ ${ }^{3}$ Kinderordination Alsergrund, Wien, Österreich \\ ${ }^{4}$ Sozialmedizinisches Zentrum Ost, Wien, Österreich \\ ${ }^{5}$ Praxis für Kinder- und Jugendheilkunde, Korneuburg, Österreich \\ ${ }^{6}$ Politische Kindermedizin, Wien, Österreich \\ ${ }^{7}$ Psychotherpeutische Praxis, Hinterbrühl, Österreich
}

\title{
Welcome? Medizinische Versorgung von Flüchtlingskindern
}

\author{
11. Jahrestagung der Politischen \\ Kindermedizin, Salzburg, \\ 10.-11. November 2017
}

Fast ein Jahr nach der für uns alle besonders eindrucksvollen Tagung zum Thema Flüchtlingsmedizin erscheint der Supplementband mit den Beiträgen der Autoren und Autorinnen. Für die Herausgeber ist dies eine Gelegenheit nachzudenken, was uns bewogen hat, die Jahrestagung zu diesem Thema auszurichten, die noch immer sehr lebendigen und aktuellen Beiträge noch einmal in ihrer noch immer nachwirkenden Präsenz zu reflektieren und einem größeren Publikum zugänglich zu machen, und auch darüber nachzudenken, was in der Zwischenzeit geschehen ist.

Der Verein Politische Kindemedizin hat sich aus einer Gruppe engagierter Menschen entwickelt, die ihr berufliches Leben der Förderung der Gesundheit der ihnen anvertrauten Kinder gewidmet haben und die ihre Unzufriedenheit mit bestimmten Bedingungen dieser Arbeit nicht verdrängen oder mit einem Achselzucken quittieren, sondern sich aktiv dafür einsetzen wollten, diese Bedingungen $\mathrm{zu}$ verbessern. Viele haben gesagt, dass dies Jammern auf hohem Niveau ist; wir sehen das anders: So lange Kinder und Jugendliche nicht nach den auch von Österreich unterzeichneten UNO-Resolutionen, nach den Bestimmungen der Bundesverfassung und der in der Sozialgesetzgebung garantierten medizinischen Versorgung, unabhängig von ihren wirtschaftlichen und sozialen Bedingungen, auf einem leistbaren Niveau behandelt werden, werden wir nicht wegschauen und dazu schweigen.

Wie bei jeder Jahrestagung der Politischen Kindermedizin, erstellen die Teilnehmer und Teilnehmerinnen eine Resolution, in der auf die noch immer bestehenden Missstände und Ungerechtigkeiten und die sich aus dem Jahrestagungsthema ergebenden besonderen Probleme hingewiesen und auch Lösungsvorschläge erarbeitet werden. Wir haben gelernt, mit dem meist positiven Echo und den tatsächlich geringen Änderungen umzugehen. Wir haben auch gelernt, dass im politischen Leben Hartnäckigkeit und der stete Tropfen imstande sind, zumindest das Bewusstsein wachzuhalten, dass nicht alles zum Besten im „besten Gesundheitssystem der Welt?" ist.

Die Beiträge befassen sich mit den Hintergründen der Flüchtlingsbewegung und den sich nicht nur aus dem
Flüchtlingsansturm 2015 ergebenden ungelösten menschlichen, wirtschaftlichen und gesundheitlichen Problemen. Die Beiträge haben sich vielfach aus dem Engagement von Menschen, die das Leid der aus ihrer Heimat unter schrecklichsten Bedingungen Vertriebenen und hier um Hilfe ersuchenden Menschen nicht kalt lässt, ergeben. Uns allen ist die große spontane Solidaritätsbewegung mit den vielen auch bei uns angekommenen Menschen, darunter auch einem großen Anteil von Kindern, die unvorstellbar Schreckliches in ihrer Heimat, auf der Flucht und auch nach ihrer Ankunft in Europa erlebt haben, in Erinnerung. Uns ist auch bewusst, wie sehr die Schwierigkeiten in der Bewältigung der Probleme dieser Menschen politisch instrumentiert wurde und eine Gegenströmung der Nichtwillkommenskultur bewirkt hat, die uns alle traurig macht.

Unvergesslich sind uns der Beitrag von Petra Ramsauer zur Realität des Kriegsgeschehens in Syrien, die fundierte Analyse von Rainer Münz zum weltweiten Problem der Flüchtlingsströme und Nathalie Simonnots bzw. Margaretha 
Malehs Bericht über das weltweite Engagement und die Arbeit der Médecins du Monde und der Médecins sans Frontières. Efi Latsoudi, die leider nicht nach Österreich kommen und uns auch keinen schriftlichen Bericht liefern konnte, hat uns in ihrem Video, das auf der Tagung gezeigt wurde, mit ihrem Optimismus gezeigt, dass auch unter widrigsten Bedingungen Menschlichkeit im Umgang mit Flüchtlingen möglich ist. Katharina Glawischnig hat die Realität der rechtlichen und finanziellen Situation von Flüchtlingen präsentiert, Nicole Grois und Regina Rath-Wacenovsky, die sich nicht nur in ihrer täglichen kinderärztlichen Arbeit für eine Standardisierung und Angleichung der Flüchtlingsmedizin an die medizinischen Standards in Österreich einsetzen, haben ihre Vorschläge präzisiert; Andrea Dixius hat das von ihr und Eva Möhler im Saarland entwickelte und angewandte START Modell zur niederschwelligen Behandlung von traumatisierten Flüchtlingskindern vorgestellt. Monika Culen hat das ebenfalls weltweite Engagement der Roten Nasen Clowns, Wissen mit einem bisschen $\mathrm{Hu}-$ mor in die trostlose Flüchtlingswelt zu bringen, eindrucksvoll dargestellt.

Die ebenfalls traditionelle RoundTable-Podiumsdiskussion hat auch bei den beteiligten Politikern Nachdenken bewirkt. Ein Teil von ihnen ist vielleicht in der Lage, etwas zu verändern. Erfolgreiche österreichische Modelle wurden von Ferdinand Sator und Daneta Memisevic (Integrationshaus), Sonja Brauner (Hemajat), Johanna Sengschmid (RMS Refugee Midwife Service) und Maria Kletecka-Pulker (Videodolmetsch) vorgestellt. Sie beruhen auf der Initiative Weniger, die durch ihre Beharrlichkeit und Zielstrebigkeit aber Unglaubliches bewirken konnten und weiter bewirken.

Möge dem vorgestellten Band, der nicht ohne das Engagement aller Beteiligten, der Mitveranstalter, der Österreichischen Gesellschaft für Kinder- und Jugendheilkunde, der Gesundheit Österreich $\mathrm{GmbH}$ und dem Hauptverband der Österreichischen Sozialversicherungsträger, der unglaublichen Unterstützung des Springer Verlags und aller Teilnehmer und Teilnehmerinnen zustande gekommen wäre, Erfolg im Sinn von
Bewusstseinsbildung und der Verbreitung von Wissen beschieden sein! Die Herausgeber

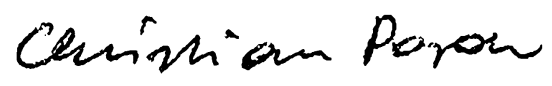

Christian Popow

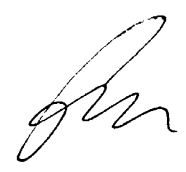

Nicole Grois

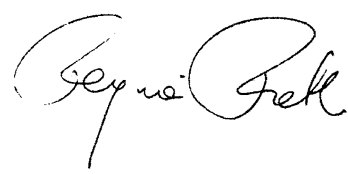

Regina Rath-Wacenovsky

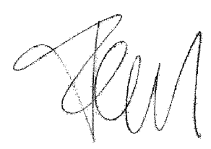

Ferdinand Sator

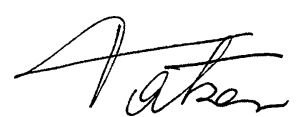

Ernst Tatzer

\section{Korrespondenzadresse}

Univ.-Prof. Dr. Christian Popow

Universitätsklinik für Kinder- und Jugendpsychiatrie, Medizinische Universität Wien

Währinger Gürtel 18-20, 1090 Wien, Österreich christian.popow@meduniwien.ac.at

Interessenkonflikt. C. Popow, N. Grois, R. RathWacenovsky, F. Sator und E. Tatzer geben an, dass kein Interessenkonflikt besteht. 